\title{
Tratamiento exitoso de una fusariosis diseminada en un paciente neutropénico febril con terapia antifúngica combinada de voriconazol más anfotericina $\mathrm{B}$ deoxicolato
}

\author{
Roberto Olivares', Mario Luppi' y M. Cristina Díaz
}

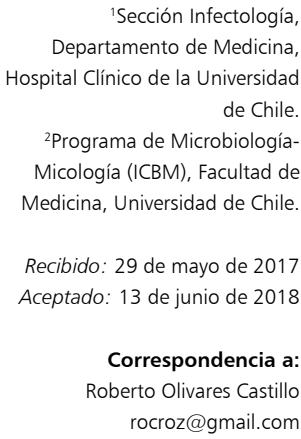

Castillo rocroz@gmail.com

$$
\text { gía- }
$$
by Fusarium verticillioides during chemotherapy-induced neutropenia. He was successfully treated only after combination therapy with voriconazole plus amphotericin B deoxycolate was used, but not when these compounds were used in an isolated form.

Key words: Fusarium; disseminated fusariosis; combined antifungal therapy; invasive fungal infection.

\section{L}

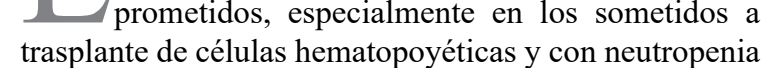
secundaria celulas hematopoyéticas y con neutropenia secundaria a quimioterapia por neoplasias hematológicas ${ }^{1}$. Este aumento en parte al amplio uso de compuestos antifúngicos ya sea en forma profiláctica o terapéutica durante los períodos prolongados de neutropenia, hecho frecuente en este tipo de pacientes y que ha permitido la selección de especies de hongos más resistentes ${ }^{2,3}$. La fusariosis diseminada ha emergido en algunos centros oncológicos como la segunda causa más frecuente de infección por hongos filamentosos después de la aspergilosis invasora ${ }^{2,4-6}$. Además, la mortalidad asociada a esta infección sigue siendo alta, debido al intenso inmunocompromiso de los pacientes afectados y porque Fusarium spp tiende a ser resistente a varios de los antifúngicos disponibles ${ }^{2,5-7}$. Un análisis de 84 pacientes con enfermedades hematológicas mostró tasas de sobrevida a los 30 y 90 días después del diagnóstico de 50 y $21 \%$; respectivamente ${ }^{7}$. Mientras que una serie más reciente de 26 casos con fusariosis, reportó una mortalidad de 50\% para el grupo de pacientes con fusariosis diseminada ${ }^{8}$. Fusarium solani se describe como la especie más frecuentemente aislada, la más virulenta, resistente y con mayor mortalidad del género, comparado con otras como Fusarium verticillioides, Fusarium oxysporum, Fusarium moniliforme, etc. ${ }^{2,6}$.

Paciente de 16 años de edad con diagnóstico de una leucemia linfoblástica aguda en recaída el año 2004. Inició un ciclo de quimioterapia de rescate, (día 0) evolucionando con una aplasia medular y una neutropenia febril grave (recuento cero de neutrófilos). Se tomaron hemocultivos y se inició tratamiento antibacteriano empírico con imipenem y vancomicina. Los hemocultivos resultaron negativos. 
Al día +35 de evolución post-quimioterapia se informaron dos hemocultivos positivos para Fusarium spp., (por sistema automatizado de hemocultivos, BacT/Alert ${ }^{\circledR}$, a las $72 \mathrm{~h}$ de incubación), el que posteriormente fue identificado como Fusarium verticillioides por sus características morfológicas macro y microscópicas.

La identificación se basó en el rápido crecimiento de las colonias con un abundante micelio aéreo de color blanco y púrpura (Figura 3). El examen microscópico reveló conidióforos naciendo lateralmente de las hifas, escasamente ramificados, monofiadílicos. También se observaron acroconidias delicadas, delgadas y septadas junto a microconidias ovoídeas que nacían de monofiálides en cadenas sin clamidoconidias (Figura 4). El diagnóstico micológico de especie se realizó en el Laboratorio de Micología del Instituto de Ciencias Biomédicas de la Facultad de Medicina de la Universidad de Chile y es consistente con lo descrito en la literatura ${ }^{13}$. No se realizaron pruebas diagnósticas de tipo molecular ni para determinar susceptibilidad a antifúngicos por no encontrarse disponibles.

Debido a la identificación de $F$. verticillioides como el agente causal y la falta de respuesta clínica a anfotericina B, se decidió aumentar la dosis a $100 \mathrm{mg} /$ día y asociar voriconazol (dosis de carga i.v. $6 \mathrm{mg} / \mathrm{kg}$, dos veces al día y luego una dosis de mantención de $4 \mathrm{mg} / \mathrm{kg}$, dos veces al día, vía oral).

Una vez iniciada la terapia combinada, se evidenció una respuesta favorable y rápida, con una disminución significativa de las mialgias y artralgias y desaparición de la fiebre a partir del segundo día de tratamiento combinado. El paciente se mantuvo todavía en aplasia, la que

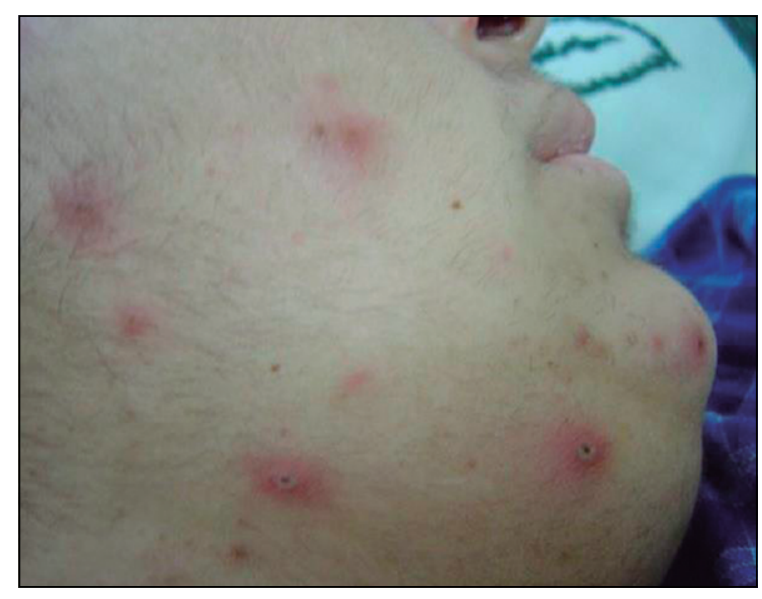

Figura 1. Lesiones cutáneas tipo ectima gangrenoso.

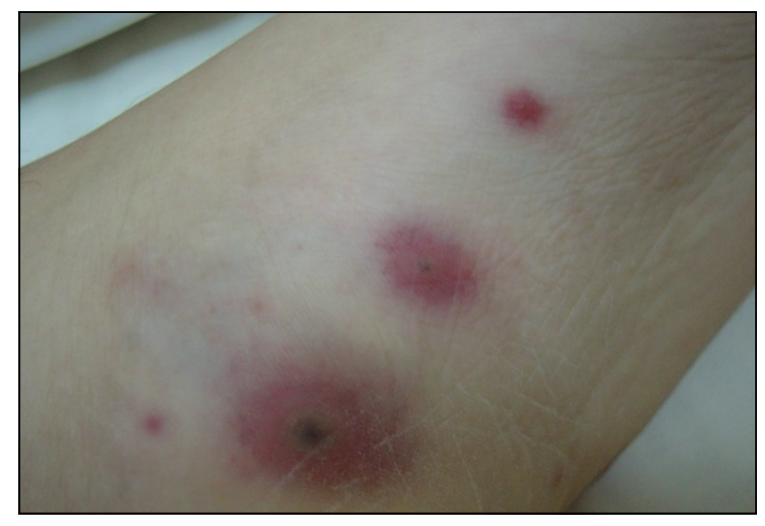

Figura 2. Lesiones cutáneas tipo ectima gangrenoso.

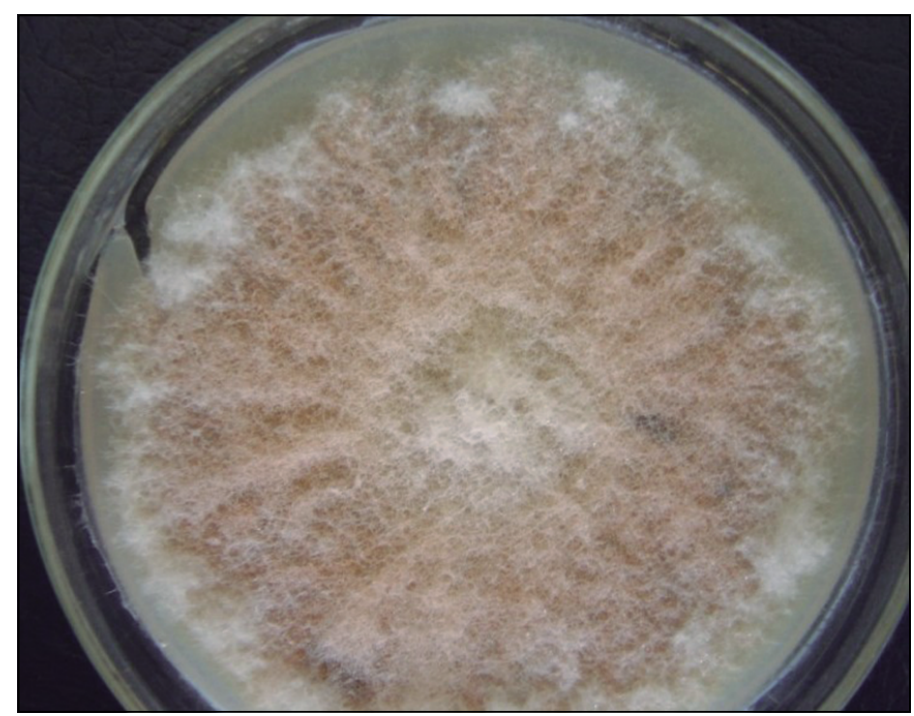

Figura 3. Fusarium verticilioides en cultivo.

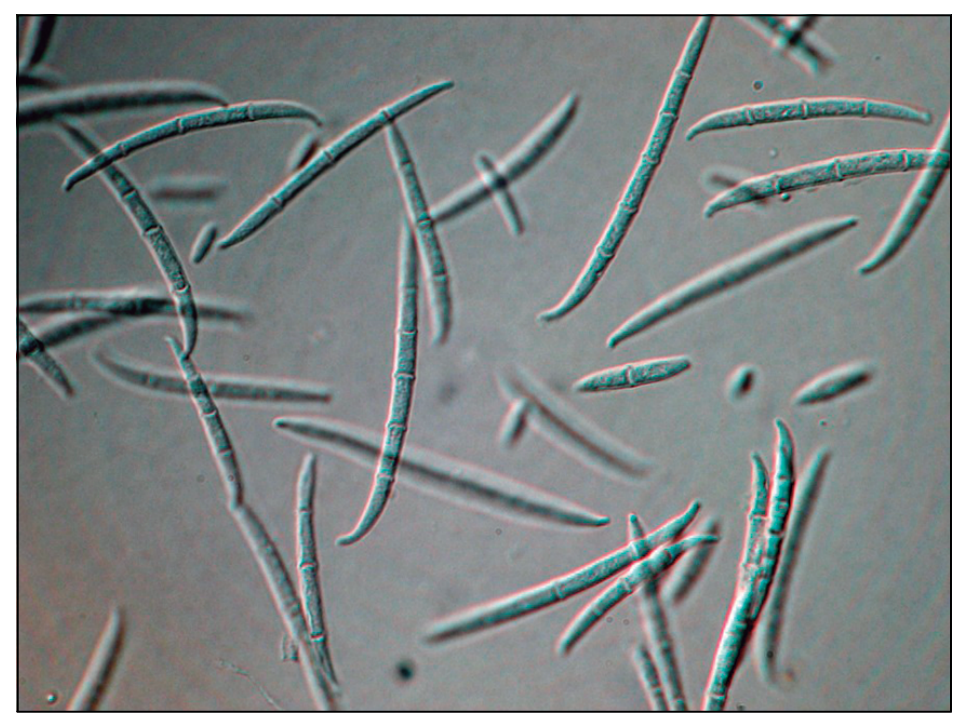

Figura 4. Macroconidios con morfología característica tipo canoa o banana. 
comenzó a recuperarse desde el cuarto día de iniciada la terapia antifúngica combinada (día +39$)$.

El informe de la biopsia cutánea fue compatible con una infección por un hongo hialino y filamentoso. El cultivo de la lesión cutánea fue negativo.

Durante todo el período de neutropenia el paciente recibió factor estimulante de colonias granulocíticas.

Debido a la excelente respuesta, se decidió suspender anfotericina $\mathrm{B}$, habiendo acumulado una dosis total de 1,7 g y llevando, con seis días de terapia combinada, manteniendo voriconazol vía oral $300 \mathrm{mg}$, dos veces al día. No hubo disponibilidad de medir niveles plasmáticos de voriconazol.

Cuatro días después de suspendida la anfotericina B, reapareció la fiebre y nuevas lesiones cutáneas similares a las ya descritas. El paciente además presentó rinorrea purulenta por primera vez. Se tomó cultivo de secreción nasal que también resultó positivo para Fusarium verticillioides. Ante estos hallazgos se decidió reiniciar anfotericina B con lo que nuevamente bajó la fiebre y las lesiones cutáneas dejaron de aparecer.

El tratamiento con anfotericina B se suspendió definitivamente el día +69 , después de 59 días de terapia, completando a esa fecha $3 \mathrm{~g}$ en total de anfotericina y 41 días de voriconazol. El paciente se mantuvo afebril, sin neutropenia y con sus lesiones en fase de cicatrización, sin reaparición de nuevas lesiones cutáneas. Los hemocultivos de control fueron negativos y las TC de tórax y de cavidades paranasales fueron ambos normales.

Los eventos adversos relacionados con los antifúngicos utilizados fueron mínimos y bien tolerados. En relación a voriconazol, el paciente refirió presentar alteraciones visuales (visión en color verde), en las primeras $48 \mathrm{~h}$ de tratamiento. La función renal no se alteró durante el uso de anfotericina B y hubo buena tolerancia a la infusión (administración en seis horas).

El paciente fue dado de alta, al día +73 post quimioterapia, con voriconazol vía oral $300 \mathrm{mg}$, dos veces al día, el que mantuvo por tres semanas más; sin embargo, debió ser suspendido ya que se consideró fuera del alcance terapéutico por recurrencia de su leucemia. El paciente falleció dos meses después debido a una hemorragia subaracnoidea.

\section{Discusión}

Fusarium spp. es un hongo filamentoso saprófito que pertenece al grupo de los agentes productores de hialohifomicosis. Es considerado un hongo emergente, dado que la forma invasora de esta enfermedad ha ido en aumento en pacientes inmunocomprometidos en los últimos años. Los más afectados son los pacientes con neoplasias hematológicas y neutropenia prolongada inducida por quimioterapia y los sometidos a trasplantes de células hematopoyéticas ${ }^{1}$. Nuestro paciente pertenece al primer grupo mencionado y evolucionó con un cuadro clínico que está frecuentemente descrito en la fusariosis invasora en su forma diseminada, la que compromete frecuentemente piel, pulmones, cavidades paranasales y que con frecuencia tienen hemocultivos positivos ${ }^{2,3,4,6}$.

La mortalidad de esta infección oportunista continúa siendo alta y es críticamente dependiente de la recuperación de los neutrófilos, todo esto sumado a que el tratamiento tradicional utilizado en el pasado para esta micosis invasora, la anfotericina $\mathrm{B}$ deoxicolato, ha mostrado resultados muy limitados. Además, Fusarium spp. es resistente a muchos compuestos antifúngicos, haciendo que el pronóstico de esta enfermedad fuera ominoso ${ }^{2,5,6,7}$.

Voriconazol ha demostrado ser activo contra Fusarium spp. lo que ha mejorado el pronóstico en estos pacientes en numerosas experiencias clínicas y se ha transformado en la terapia de primera línea para este tipo de infección fúngica invasora ${ }^{9-12,14,18}$.

Nuestro paciente corresponde a una fusariosis diseminada diagnosticada en nuestro hospital y fue el primer caso en que tuvimos la oportunidad de utilizar la terapia combinada de voriconazol y anfotericina B deoxicolato. El paciente tuvo una presentación y evolución clínica de acuerdo a lo descrito en una fusariosis invasora: neutropenia prolongada, fiebre persistente a pesar de terapia antibacteriana de amplio espectro, desarrollo de múltiples lesiones cutáneas, la mayoría de ellas tipo ectima gangrenoso, compromiso de senos paranasales y hemocultivos positivos $^{15,16}$. No obstante, este caso presenta algunos aspectos de interés que nos parece importante discutir.

En primer lugar, la respuesta clínica fue exitosa cuando la terapia antifúngica se utilizó en forma combinada. La fiebre remitió, las lesiones cutáneas mejoraron y no aparecieron nuevas lesiones. Esto ocurrió incluso antes de la recuperación de la neutropenia. En nuestra experiencia, solo el tratamiento asociado con voriconazol y anfotericina $\mathrm{B}$ deoxicolato permitió el control de la infección y la recuperación del paciente durante la neutropenia.

En las dos ocasiones que el paciente estuvo con monoterapia antifúngica, ésta se asoció a una mala respuesta clínica y/o una recurrencia de los síntomas y signos. Al inicio de la terapia, la anfotericina no logró controlar la infección. Posteriormente, una vez que el paciente mejoró clínicamente, anfotericina fue suspendida y recibió solo voriconazol, presentó precozmente una reactivación del cuadro clínico. Solo después de la reintroducción de anfotericina y reiniciando la terapia antifúngica combinada, se objetivó una respuesta clínica favorable, con caída de la fiebre y desaparición nuevamente de las lesiones cutáneas.

El uso de terapia combinada en una fusariosis diseminada está en concordancia con lo reportado en la literatura especializada a partir del año 2001. Un estudio analizó en 
forma retrospectiva dos períodos de tratamiento, desde el año 1985 al 2001 (período 1) y de 2001 al 2011 (período 2). El tratamiento primario con anfotericina $B$ deoxicolato fue más frecuente en el período 1, mientras voriconazol y las terapias combinadas fueron más frecuentes en el período 2. Las probabilidades de sobrevida fueron $22 \mathrm{y}$ $43 \%$ en los períodos 1 y 2 ; respectivamente ${ }^{17}$.

Sin embargo, no existen ensayos clínicos aleatorios que definan el tratamiento óptimo para la fusariosis diseminada, sino más bien solo series de $\operatorname{casos}^{18,19}$. Uno de éstos describe 20 casos de fusariosis invasora con terapia combinada durante 10 años; 14 respondieron favorablemente y siete lo hicieron antes de la resolución de la neutropenia ${ }^{18}$, tal como ocurrió en nuestro caso.

En otra revisión de 65 casos de fusariosis invasora, $70 \%$ recibió terapia antifúngica, de los cuales 24 recibieron monoterapia (la mayoría con voriconazol) y 22 , terapia combinada (siendo la más frecuente voriconazol más anfotericina liposomal). La tasa de sobrevida a los 90 días fue de $44 \%$ (lo que implica una mejoría respecto a datos históricos) ${ }^{19}$.

Más recientemente, reportes de casos han documentado éxito con terapia combinada (voriconazol más anfotericina liposomal). No obstante, la evidencia es mixta, con otras series en que no se mostró beneficio e incluso con mayor mortalidad $^{8,20}$.

La revisión más extensa publicada hasta el momento, con 223 casos diagnosticados por casi tres décadas, mostró una probabilidad de sobrevida a los 90 días, según el tipo de terapia, de $60 \%$ para voriconazol, $53 \%$ para la formulación lipídica de anfotericina y solo $28 \%$ para anfotericina B deoxicolato ${ }^{17,21}$.

Por un tema de disponibilidad y costo en ese momento, no se utilizó anfotericina liposomal, que es uno de los antifúngicos de elección en estos casos. Incluso, actualmente se desaconseja su uso en fusariosis invasora, dado que se ha asociado a peores desenlaces ${ }^{19,21}$. En este caso, el uso de anfotericina $\mathrm{B}$ deoxicolato tuvo un impacto positivo en la evolución y resolución del cuadro cuando se combinó con otro antifúngico activo contra Fusarium spp. Esto a su vez, podría explicarse por la especie de Fusarium aislado en este caso.

Fusarium verticillioides se ha descrito como una especie con una mejor tasa de respuesta al tratamiento antifúngico, debido a una mayor susceptibilidad y probablemente una menor virulencia que $F$. solani, confiriéndole un mejor pronóstico a estos pacientes ${ }^{18,21}$.

Consideramos este hecho clave en la respuesta a la terapia antifúngica combinada utilizada en nuestro caso.

Los efectos adversos asociados con el uso de anfoteri- cina deoxicolato no fueron significativos en este paciente (no presentó falla renal). Esto en parte se explica por ser joven y por no haber recibido otros fármacos nefrotóxicos.

Voriconazol es considerado un medicamento de alto costo en nuestro medio. La excelente biodisponibilidad de este compuesto nos permitió un cambio precoz a terapia oral, permitiendo una contención de costos sin renunciar a un desenlace clínico favorable.

Dentro de las limitaciones de esta comunicación podemos mencionar en primer lugar que dado que es un caso antiguo, el diagnóstico micológico fue hecho en base a técnicas de estudio tradicionales, no habiendo disponibilidad en ese entonces de técnicas de biología molecular que permitieran un diagnóstico más preciso y específico. Además tampoco se contó con análisis de susceptibilidad para poder orientar de una mejor manera la terapia antifúngica.

Un segundo factor a considerar es que no se dispuso de niveles de voriconazol para monitorizar la concentración plasmática del fármaco. Voriconazol presenta una farmacocinética no lineal, con grandes variaciones en su concentración plasmática tanto a nivel individual como en distintos pacientes. Se ha observado que en terapias prolongadas se puede producir una autoinducción del citocromo p450, resultando en una disminución de las concentraciones plasmáticas de voriconazol. Esto podría explicar por qué nuestro paciente no respondió clínicamente cuando recibió monoterapia con voriconazol ${ }^{22}$.

En conclusión, la fusariosis diseminada es una infección fúngica que debe ser sospechada en pacientes con neoplasias hematológicas y neutropenia intensa y persistente. El desenlace favorable de este caso pudo estar relacionado con el uso de tratamiento antifúngico combinado y la presencia de una especie de Fusarium menos agresiva como $F$. verticillioides.

El manejo debe ir dirigido a intentar disminuir la inmunosupresión y recuperar la neutropenia asociado al uso de terapia antifúngica combinada para lograr un mejor desenlace.

\section{Resumen}

Presentamos el caso clínico de un paciente con una leucemia linfoblástica aguda (LLA) que desarrolló una fusariosis diseminada por Fusarium verticillioides durante un episodio prolongado de neutropenia febril post quimioterapia. Fue exitosamente tratado cuando se usó terapia combinada de voriconazol más anfotericina B deoxicolato. 


\section{Referencias bibliográficas}

1.- Garnica M, da Cunha M O, Portugal R, Maiolino A, Colombo A L, Nucci M. Risk factors for invasive fusariosis in patients with acute myeloid leukemia and in hematopoietic cell transplant recipients. Clin Infect Dis 2015; 60: 875-80. doi: 10.1093/cid/ ciu947.

2.- Lionakis M S, Kontoyiannis D P. Fusarium infections in critically ill patients. Semin Respir Crit Care Med 2004; 25: 159-69.

3.- Baddley J W, Stroud T P, Salzman D, Pappas P $\mathrm{G}$. Invasive mold infections in allogeneic bone marrow transplant recipients. Clin Infect Dis 2001; 32: 1319-24.

4.- Boutati E, Anaissie E J. Fusarium, a significant emerging pathogen in patients with hematologic malignancy: ten years' experience at a cancer center and implications for management. Blood 1997; 90: 999-1008.

5.- Nucci M. Emerging moulds: Fusarium, Scedosporium and Zygomycetes in transplant recipients. Curr Opin Infect Dis 2003; 16: 607 12.

6.- Nucci M, Anaissie E. Fusarium infections in immunocompromised patients. Clin Microbiol Rev 2007; 20: 695-704.

7.- Nucci M, Anaissie E, Queiroz-Telles F, Martins C A, Trabasso P, Solza C, et al. Outcome predictors of 84 patients with hematologic malignancies and Fusarium infection. Cancer 2003; 98: 315-9.

8.- Muhammed M, Anagnostou T, Desalermos A, Kourkoumpetis T, Carneiro H, Glavis-Bloom J, et al. Fusarium infection: report of 26 cases and review of 97 cases from the literature.
Medicine 2013; 92: 305-316. doi: 10.1097/ MD.0000000000000008.

9.- Cosigny S, Dhedin N, Datry A, Choquet S, Leblond V, Chosidow O. Successful voriconazole treatment of disseminated Fusarium infection in an immunocompromised patient. Clin Infect Dis 2003; 37: 311-3.

10.- Durand-Joly I, Alfandari S, Benchikh Z, Rodrigue M, Espinel-Ingroff A, Catteau B, et al. Successful outcome of disseminated Fusarium infection with skin localization treated with voriconazole and amphotericin B-lipid complex in a patient with acute leukemia. J Clin Microbiol 2003; 41: 4898-900.

11.- Rothe A, Seibold M, Hoppe T, Seifert H, Engert A, Caspar C, et al. Combination therapy of disseminated Fusarium oxysporum infection with terbinafine and amphotericin B. Ann Hematol 2004; 83: 394-97.

12.- Letscher-Bru V, Campos F, Waller J, Randriamahazaka R, Candolfi E, Herbrecht R. Successful outcome of treatment of a disseminated infection due to Fusarium dimerum in a leukemia patient. J Clin Microbiol 2002; 40: 1100-2

13.- Tapia C, Amaro J. Género Fusarium. Rev Chilena Infectol 2014; 31: 85-6. doi: 10.4067/ S0716-10182014000100012.

14.- Johnson L B, Kauffman C A. Voriconazole: a new triazole antifungal agent. Clin Infect Dis 2003; 36: 630-7.

15.- Walsh T, Groll A, Hiemenz J, Fleming R, Roilides E, Anaissie E. Infections due to emerging and uncommon medically important fungal pathogens. Clin Microbiol Infect 2004; 10 (Suppl 1): 48-66.
16.- Nucci M, Anaissie E. Cutaneous infection by Fusarium species in healthy and inmunocompromised hosts: implications for diagnosis and management. Clin Infect Dis 2002; 35: 909-20.

17.- Nucci M, Marr K A, Vehreschild M J, de Souza C, Velasco E, Cappellano P, et al. Improvement in the outcome of invasive fusariosis in the last decade. Clin Microbiol Infect 2014; 20: 580-5. doi: 10.1111/1469-0691.12409.

18.- Guarro J. Fusariosis, a complex infection caused by a high diversity of fungal species refractory to treatment. Eur J Clin Microbiol Infect Dis 2013; 32: 1491-500. doi: 10.1007/ s10096-013-1924-7.

19.- Horn D L, Freifeld A G, Schuster M G, Azie N E, Franks B, Kauffman C. Treatment and outcomes of invasive fusariosis: review of 65 cases from the PATH Alliance registry. Mycoses 2014; 57(11): 652-8. doi: 10.1111/ myc. 12212.

20.- Blyth C, Gilroy N M, Guy S D, Chambers S T, Cheong E Y, Gottlieb T, et al. Consensus guidelines for the treatment of invasive mould infections in haematological malignancy and haemopoietic stem cell transplantation, 2014. Intern Med J 2014; 44: 1333-49. doi: 10.1111/ imj. 12598 .

21.- Nucci M, Nouér S, Capone D, Anaissie E, Nucci M. Fusariosis. Semin Respir Crit Care 2015; 36: 706-14. doi: 10.1055/s-00351562897.

22.- Malani A N , Kerr L E, Kauffman C A. Voriconazole: how to use this antifungal agent and what to expect. Semin Respir Crit Care Med 2015; 36: 786-95. doi: 10.1055/s-00351562903. 